OPEN ACCESS

Edited by:

Ruth Sapir-Pichhadze, McGill University Health Centre,

Canada

Reviewed by:

Stephen Alexander,

The University of Sydney, Australia

Javier Carbone,

Gregorio Marañón Hospital, Spain

*Correspondence:

Andreas Heinzel

andreas.heinzel@meduniwien.ac.at

Rainer Oberbauer

rainer.oberbauer@meduniwien.ac.at

${ }^{\dagger}$ These authors have contributed equally to this work

Specialty section:

This article was submitted to Alloimmunity and Transplantation,

a section of the journal

Frontiers in Immunology

Received: 25 December 2021 Accepted: 03 February 2022

Published: 25 February 2022

Citation:

Aschauer C, Jelencsics K, Hu K, Gregorich M, Reindl-Schwaighofer R, Wenda S, Wekerle T, Heinzel A and

Oberbauer $R$ (2022) Effects of

Reduced-Dose Anti-Human TLymphocyte Globulin on Overall and

Donor-Specific T-Cell Repertoire Reconstitution in Sensitized Kidney

Transplant Recipients.

Front. Immunol. 13:843452. doi: 10.3389/fimmu.2022.843452

\section{Effects of Reduced-Dose Anti-Human T-Lymphocyte Globulin on Overall and Donor-Specific T-Cell Repertoire Reconstitution in Sensitized Kidney Transplant Recipients}

\author{
Constantin Aschauer ${ }^{1+}$, Kira Jelencsics ${ }^{1+}$, Karin $\mathrm{Hu}^{1}$, Mariella Gregorich ${ }^{1,2}$, \\ Roman Reindl-Schwaighofer ${ }^{1}$, Sabine Wenda ${ }^{3}$, Thomas Wekerle ${ }^{4}$, \\ Andreas Heinzel ${ }^{1 *}$ and Rainer Oberbauer ${ }^{1 *}$

\begin{abstract}
1 Division of Nephrology and Dialysis, Department of Medicine III, Medical University of Vienna, Vienna, Austria, 2 Section for Austria, ${ }^{3}$ Department of Blood Group Serology and Transfusion Medicine, Medical University Vienna, Vienna, Austria, ${ }^{4}$ Division of Transplantation, Department of General Surgery, Medical University of Vienna, Vienna, Austria
\end{abstract} \\ Clinical Biometrics, Center for Medical Statistics, Informatics and Intelligent Systems, Medical University of Vienna, Vienna,
}

Background: Pre-sensitized kidney transplant recipients have a higher risk for rejection following kidney transplantation and therefore receive lymphodepletional induction therapy with anti-human T-lymphocyte globulin (ATLG) whereas non-sensitized patients are induced in many centers with basiliximab. The time course of lymphocyte reconstitution with regard to the overall and donor-reactive T-cell receptor (TCR) specificity remains elusive.

Methods/Design: Five kidney transplant recipients receiving a $1.5-\mathrm{mg} / \mathrm{kg}$ ATLG induction therapy over 7 days and five patients with $2 \times 20 \mathrm{mg}$ basiliximab induction therapy were longitudinally monitored. Peripheral mononuclear cells were sampled pretransplant and within 1, 3, and 12 months after transplantation, and their overall and donor-reactive TCRs were determined by next-generation sequencing of the TCR beta CDR3 region. Overall TCR repertoire diversity, turnover, and donor specificity were assessed at all timepoints.

Results: We observed an increase in the donor-reactive TCR repertoire after transplantation in patients, independent of lymphocyte counts or induction therapy. Donor-reactive CD4 T-cell frequency in the ATLG group increased from 1.14\% + -0.63 to $2.03 \%+-1.09$ and from $0.93 \%+-0.63$ to $1.82 \%+-1.17$ in the basiliximab group in the first month. Diversity measurements of the entire T-cell repertoire and repertoire turnover showed no statistical difference between the two induction therapies. The difference in mean clonality between groups was 0.03 and 0.07 pre-transplant in the CD4 and CD8 fractions, respectively, and was not different over time $(C D 4: F(1.45,11.6)=0.64 p=$ 0.496; CD8: $F(3,24)=0.60 p=0.620$ ). The mean difference in $R 20$, a metric for immune dominance, between groups was -0.006 in CD4 and 0.001 in CD8 T-cells and not 
statistically different between the groups and subsequent timepoints $(C D 4: F(3,24)=0.85$ $p=0.479$; CD8: $F(1.19,9.52)=0.79 p=0.418)$.

Conclusion: Reduced-dose ATLG induction therapy led to an initial lymphodepletion followed by an increase in the percentage of donor-reactive T-cells after transplantation similar to basiliximab induction therapy. Furthermore, reduced-dose ATLG did not change the overall TCR repertoire in terms of a narrowed or skewed TCR repertoire after immune reconstitution, comparable to non-depletional induction therapy.

Keywords: kidney transplantation, immune reconstitution, anti-T lymphocyte globulin, allorepertoire, T-cell receptor (TCR) repertoire, lymphocyte depletion therapy, alloreactivity

\section{BACKGROUND}

Prevention of rejection and achievement long-lasting graft function represents one of the major challenges after kidney transplantation. Especially highly immunized individuals are at a higher risk for alloimmunity, and therefore medical immunosuppression is increased to eradicate preexisting donor-reactive lymphocytes and reduce donor-specific antihuman leukocyte antigen (HLA) antibodies (DSAs) in these recipients $(1-3)$.

Patients at risk receive a lymphodepletional induction therapy in most transplant centers such as rabbit anti-human T-lymphocyte globulin (ATLG) or another lymphodepletional agent combined with plasma exchange or immunoadsorption (3).

Dosing for ATLG is heterogeneous in clinical practice as high dosing leads to a profound long-lasting lymphopenia with functional impaired immune cells following reconstitution and increases the incidence of infections and cancer $(4,5)$. Therefore, lower dosing regimens have been evaluated to reduce side effects without increased risk of rejecting the allograft (6-8).

ATLG provides multifaceted immunomodulation, including T-cell depletion in blood and peripheral lymphoid tissues, induction of apoptosis in B-cell lineages, interference with dendritic cell functional properties, induction of regulatory $\mathrm{T}$ cells and NKT cells, and skewed immune repertoire and impaired T-cell function after reconstitution (9-11).

Before the upcoming of new techniques and tools to define donor-specific T-cells, studies have been focusing mostly on the phenotype and functionality but to a lesser extent on the specificity of the reconstituted T-cells $(5,11-18)$.

Cherkasky et al. first observed a marked and prolonged donor hyporesponsiveness with minimal effects on non-donor responses after Thymoglobuline ${ }^{\circledR}$ (ATG) induction using ELISPOT assays of the reconstituted T-cell repertoire (19). The

Abbreviations: ANOVA, analysis of variance; ATG, anti-thymocyte globulin; ATLG, anti-T-lymphocyte globulin; CDR3, complementarity determining region 3; CFSE, carboxyfluorescein succinimidyl ester; CMV, cytomegalovirus; DSA, donor-specific anti-HLA antibody; EBV, Epstein-Barr virus; ELISPOT, enzymelinked immunospot; FACS, fluorescence-activated cell sorting; MFI, mean fluorescence intensity; MIGEC, Molecular Identifier Guided Error Correction pipeline; MLR, mixed lymphocyte reaction; NK cells, natural killer cells; PBS, phosphate-buffered saline; TAC, tacrolimus; TCR, T-cell receptor; UMI, unique molecular identifier; V(D)J, Variable, Diversity, Joining; VPD, Violet proliferation dye. underlying mechanisms for this donor hyporesponsiveness were not elucidated, but a promotion of regulatory $\mathrm{T}$-cells that are specific for donor antigens following lymphodepletion have been hypothesized.

These findings support the hypothesis that the ATG effect is the result not only from immunodepletion but also from the induction of T-cells that control allogeneic immune responses.

Improvements in next-generation sequencing (NGS) technologies have made it possible to characterize the overall and donor-reactive T-cell repertoire by $\mathrm{T}$-cell receptor (TCR) sequencing of the circulating T-cells $(20,21)$. In addition, it is possible to specifically define the donor-reactive TCR repertoire pre-transplant and to subsequently track the donor-reactive TCRs after transplantation (22-25).

Recently, we were able to prove the enrichment of these predefined donor-reactive T-cells not only in the circulating TCR repertoire but also in the rejecting kidney allograft, underlining the immunological importance of these cells in non-sensitized kidney transplant recipients (23).

In this study, we sought to describe the effects of reduced-dose lymphodepletional therapy compared to basiliximab induction therapy and the reconstitution of the overall and donor-reactive TCR repertoire in kidney transplant recipients. We aimed not only to characterize lymphocyte reconstitution on a phenotype level but also to describe the TCR repertoire in a reduced-dose ATLG regimen making it possible to evaluate shifts in the shape of the immune repertoire and to also detect the reappearance of donor-reactive $\mathrm{T}$ cells following reduced lymphodepletional induction therapy.

\section{METHODS/DESIGN}

\section{Subjects}

Five pre-sensitized kidney transplant recipients, defined as the presence of suspected DSAs and five recipients without preformed DSAs, were included prior to transplantation. The included subjects did not experience a cellular rejection episode within the observation time. However, patient R294 in the ATLG group required hospital admission for urinary tract infection (at 1.5 and 4 months post-transplant) and COVID-19 infection (at 6 months post-transplant) during the follow-up period. Sampling timepoints for this patient did not coincide with hospital admissions. Pre-transplant DSA positivity was defined as a 
mean fluorescence intensity (MFI) of $>2,000$. With the exception of patient R192 who experienced a reoccurrence of a preformed DSA at high titers, only low-titer DSAs or no DSAs became detectable in the remaining patients. High-resolution HLA typing for all recipients and donors was performed as part of the clinical routine by NGS for HLA-A, B, C, DRB1, DRB3/4/5, DQA1, DQB1, and DPB1 (26). HLA antibody detection was also performed as part of the clinical routine using the Luminex Single Antigen bead assay for class I and class II (LABScreen Single Antigen, Thermo Fisher Scientific Inc., Waltham, MA). Detailed patient characteristics are provided in Table 1, and high-resolution HLA typings and DSA titers are available in Supplementary Tables $\mathbf{1}$ and 2, respectively. Recipient and donor peripheral mononuclear cells (PBMCs) were collected prior and within the first month as well as 3 and 12 months after transplantation and cryopreserved until analysis. The exact sampling timepoints for each patient are provided in Supplementary Table 3.

Pre-sensitized recipients received an induction therapy with rabbit ATLG (Grafalon ${ }^{\circledR}$ ) at a dose of $1.5 \mathrm{mg} / \mathrm{kg}$ for 7 days and concomitant immunoadsorption with GAM-146 peptide $\left(\right.$ Globaffin ${ }^{\circledR}$ ) columns. Induction therapy for non-sensitized recipients was uniformly basiliximab (Simulect ${ }^{\circledR}$ ). This reduced ATLG dosage for induction therapy in DSA-positive patients followed our center policy and is equally effective in prevention of acute cellular rejection when compared to higher ATLG dosing (27). Further immunosuppression was uniformly corticosteroids, mycophenolate-mofetil, and tacrolimus (TAC). TAC trough levels for all patients throughout the observation period are provided in

\section{Supplementary Table 4.}

Mixed lymphocyte reaction (MLR) and RNA-based TCR sequencing were performed for all included patients. Additional phenotypical characterization of T-cells by FACS was performed for the group of patients receiving ATLG induction. The TCR repertoire was compared at the four defined timepoints between these two groups of induction therapy. Institutional ethics committee approval (EC NB: 267/2011) was obtained for all aspects of the study, and all study participants were included after signed informed consent prior to transplantation.

\section{Defining the T-Cell Repertoire}

\section{Phenotypic Characterization of T-Cells}

Cryopreserved PBMCs were rapidly thawed and resuspended in pre-warmed RPMI 1640 (Gibco, Grand Island, NY, USA) containing $50 \mu \mathrm{g} / \mathrm{ml}$ DNASE I (Roche, Basel, Switzerland). Subsequently, cells were collected by centrifugation, resuspended in phosphate-buffered saline (PBS) (Gibco), and stained using DURAClone IM T Cell Subset tubes (Beckman Coulter). As DURAClone tubes are designed to be used with whole blood, additional preparatory steps were necessary for the use with isolated PBMCs. Dried reagents in the DURAClone tubes were reconstituted in $50 \mu \mathrm{l} \mathrm{PBS}$ and $5 \mu \mathrm{l}$ of BV785-labeled anti-CD103 (BioLegend, San Diego, CA, USA), and $5 \mu \mathrm{l}$ of BV605-labeled anti-CD31 (BioLegend) antibodies was added as additional antibodies. $30 \mu \mathrm{l}$ of the resulting antibody mixture was used to stain $7 \times 105$ cells in a total volume of $50 \mu \mathrm{l}$. Staining was performed according to the manufacturer's instruction skipping the red blood cell lysis step.

Cells were acquired on a Cytek Aurora (Cytek Biosciences, Fremont, CA, USA), and unmixed data were reanalyzed using Kaluza (Beckman Coulter, Brea, CA, USA). Numbers for the following T-cell phenotypes were recorded: CD4 T-cells (CD3+ CD4+), CD8 T-cells (CD3+ CD8+), CD4 recent thymic emigrants (CD3+, CD4+, CD31+), CD4- and CD8-naive T-cells (CD3+, CD4+/CD8+, CCR7+ CD45RA+), CD4 and CD8 central memory T-cells (CD3+, CD4+/CD8+, CCR7+ CD45RA-), CD4 and CD8 effector memory T-cells (CD3+, CD4+/CD8+, CCR7- CD45RA-), and CD4 and CD8 effector memory $\mathrm{T}$-cells re-expressing CD45RA (CD3+, CD4+/CD8+, CCR7- CD45RA+). The gating strategy exemplified on one representative individual is provided in Supplementary Figure 1.

\section{Mixed Lymphocyte Reaction}

Mixed lymphocyte reactions were performed as previously described, reflecting $\mathrm{T}$-cell activation driven mostly by the direct pathway of allorecognition $(23,28)$. Briefly, after thawing of cryopreserved PBMCs collected prior to transplantation, MLRs were performed by plating $2 \times 10^{5}$ carboxyfluorescein

TABLE 1 | Patients baseline characteristics.

\begin{tabular}{|c|c|c|c|c|c|c|c|c|}
\hline Subject & Sex & Age at $\mathrm{Tx}$ & Cause of ESRD & MM & Type of Tx & DSA & Immunosuppressive therapy & CMV R/D \\
\hline $\mathrm{R} 154$ & Male & 55 & Unknown & $2-1-1$ & $\begin{array}{c}\text { DKD } \\
\text { 2nd Tx }\end{array}$ & Class I; Cat 2 & IAS + ATLG & $-/-$ \\
\hline R192 & Female & 60 & IgA nephritis & $1-1-0$ & DKD & Class I; Cat 3 & IAS +ATLG & $+/-$ \\
\hline R200 & Male & 60 & Diabetes type II & $1-1-1$ & DKD & Class II; Cat 3 & IAS + ATLG & $+/-$ \\
\hline R 294 & Female & 60 & Unknown & $1-1-0$ & DKD & Class II; Cat 2 & IAS + ATLG & $+/+$ \\
\hline R 327 & Male & 59 & ADPKD & $1-1-2$ & DKD & Class II; Cat 2 & $I A S+A T L G$ & $+/-$ \\
\hline $\mathrm{R} 24^{a}$ & Male & 54 & ADPKD & $1-1-0$ & LKD & No & IAS $(7 x)+\operatorname{PLEX}(1 \mathrm{x})+$ IL-2RA & $+/-$ \\
\hline $\mathrm{R} 30$ & Male & 66 & Amyloidosis & $1-1-1$ & LKD & No & IL-2RA induction & $+/+$ \\
\hline R 190 & Male & 42 & ADPKD & $1-1-1$ & DKD & No & IL-2RA induction & $-/-$ \\
\hline R202 & Female & 65 & ADPKD & $0-1-1$ & DKD & No & IL-2RA induction & $+/+$ \\
\hline $\mathrm{R} 211$ & Male & 37 & Unknown & $1-1-0$ & DKD & No & IL-2RA induction & $+/+$ \\
\hline
\end{tabular}

ADPKD, autosomal dominant polycystic kidney disease; IAS, immunoadsorption; MMF, mycophenolatmofetil; MM, HLA mismatch (A-B-DR); PLEX, plasma exchange; DKD, diseased kidney donation; $L K D$, living kidney donation.

Category 1: MFI 1000-2000; Category 2: MFI 2000-5000; Category 3: MFI 5000-10000.

${ }^{a} R 24$ had $A B O$ incompatible transplantation. 
succinimidyl ester (CFSE)-labeled (Invitrogen cat. \#C34554) transplant recipient cells and $2 \times 10^{5}$ violet proliferation dye (VPD)-labeled (BD Horizon cat. \#562158) irradiated donor cells in each well of a 96-well plate. MLR cultures were incubated at $37^{\circ} \mathrm{C}$ for 6 days and were sorted on a FACS Aria Fusion high-speed cell sorter for CD4+ CFSE ${ }^{\text {low }}$ and CD8+ CFSE $^{\text {low }}$ T-cells (BD Pharmingen, San Diego, CA, USA, cat.\# 552852; BioLegend, San Diego, CA, USA, cat.\# 317426; BD Pharmingen cat.\# 557834). Unstimulated pre- and posttransplant samples were sorted for CD4+ and CD8+ T-cells to define the bulk repertoire. The sorting of T-cell populations was followed by RNA isolation and library preparation for TCR sequencing. A schematic overview of the method for identifying donor-reactive TCRs are found in Supplementary Figure 2.

\section{TCR Repertoire Sequencing}

RNA isolation, NGS library preparation, sequencing, and bioinformatics analysis were previously described (29). In brief, lymphocytes were sorted from PBMCs, and RNA isolation was done following the original TRIzol protocol (Invitrogen, Carlsbad, CA). TCR libraries were sequenced on an Illumina NextSeq 500. Barcodes and unique molecular identifiers were processed with MIGEC (21), and for clonotype assembly MiXCR (30) was employed.

\section{Virus-Specific TCRs}

Virus-specific TCRs were identified by matching the CDR3 sequence against TCRs with known specificity available in the VDJdb database using VDJmatch (31) (https://github.com/ antigenomics/vdjmatch).

\section{Statistical Methods}

The statistical methods used to examine the repertoires with regard to donor-reactive clonotype distribution, diversity, and similarity between repertoires followed the procedure previously described in depth in (23).

\section{Repertoire Processing}

Each repertoire comprised the following information per clonotype: the CDR3 nucleotide and amino acid sequence, reads (clones), frequency, and the $\mathrm{V}, \mathrm{D}$, and $\mathrm{J}$ genes. Ambiguity correction for CD4 and CD8 T-cells was carried out by assigning ambiguous clonotypes to the phenotype with twice the number of clones. Remaining ambiguous clonotypes were removed from the repertoire, and corresponding frequency adjustments were carried out. Clonotypes were defined as donorreactive in case of a fold change equal or greater than five with regard to the reads between the unstimulated and the stimulated pre-transplant sample. This fold expansion criterion has been used in previous studies also employing MLRs for defining the donor-specific repertoire to exclude bystander activation that may occur during the later phase of the $\operatorname{MLR}(22,23)$.

Further, all T-cell repertoires were downsampled to the lowest read count within each phenotype group to avoid immediate effects of sequencing depth on the findings of the statistical analyses. Hence, all presented results are based on the mean estimates obtained from 1,000 downsampled repertoires.

\section{Diversity and Statistical Analysis}

Diversity of each repertoire was measured in terms of (i) R20, the fraction of clonotypes (ordered by decreasing frequency) which constitute $20 \%$ of the repertoire, and (ii) clonality, defined as 1-P where $\mathrm{P}$ denotes Pielous's index which is the ratio between the observed and the maximum possible Shannon's entropy (20). Continuous variables are presented as mean \pm standard deviation (SD). Differences between the ATLG and the basiliximab group were evaluated with unpaired t-tests. The paired t-test was used to determine differences in findings between different follow-up timepoints. Mixed factorial ANOVA was used for the simultaneous assessment of differences of the between-subject factor treatment and the within-subject factors of time with regard to a continuous variable (e.g., diversity). Differences were considered significant at a p-value of less than 0.05 . $\mathrm{p}$-values were not adjusted for multiple testing due to the exploratory nature of the statistical analysis. Statistical analysis and comparisons were performed with the statistical software $\mathrm{R}$ version 4.0.2 (R Foundation for Statistical Computing, Vienna, Austria).

\section{Repertoire Overlap}

Similarity analysis to compare the overlap between the $\mathrm{T}$ cell repertoires in terms of CDR3 nucleotide sequences, VJ pairing, and $\mathrm{V}$ and $\mathrm{J}$ usage included (i) the Jensen-Shannon divergence (JSD), a similarity measure quantifying the divergence between two probability distributions, and (ii) graphical assessment by circos plots visualizing the differences in frequencies of VJ parings (32).

\section{RESULTS}

\section{Overall and Donor-Reactive TCR Repertoire}

Following induction therapy after kidney transplantation, a lymphodepletion in the ATLG group reaching a mean of $0.11+$ $-0.07 \mathrm{G} / \mathrm{L}$ over the first month was observed. The lymphopenic state in our cohort lasted for at least 14 days, and lymphocyte numbers recovered to $>1 \mathrm{G} / \mathrm{L}$ in two patients at day 310 and three subjects presented with prolonged reduced lymphocyte counts throughout the observation period. Lymphocyte counts in the basiliximab group also decreased in the first month after transplantation but rose to $>1$ $\mathrm{G} / \mathrm{L}$ in all subjects in subsequent timepoints (Table 2). Graft function determined by estimated glomerular filtration rate (eGFR) at 12 months after transplantation was not different between the groups (ATLG group: $64+-18 \mathrm{ml} / \mathrm{min} / 1.73 \mathrm{~m}^{2}$, basiliximab group: $53+-12 \mathrm{ml} / \mathrm{min} / 1.73 \mathrm{~m}^{2} ; \mathrm{p}=0.23$ ).

The absolute number of detected TCR clonotypes after transplantation via NGS sequencing of the TCR beta chain was comparable to pre-transplant in both groups at all timepoints and ranged from 26,328 to 107,735 for CD4 and 7,184 to 87,533 for CD8 T-cells. The numbers of clonotypes remaining after downsampling are shown in Figure 1. The donor-reactive repertoire defined following MLR revealed a lower number of clonotypes than the unstimulated repertoires in both cohorts, as expected. Detailed numbers of clonotypes and clones are reported in the Supplementary Tables 5 and $\mathbf{6}$. 
TABLE 2 | Time-specific distribution of lymphocyte numbers.

\begin{tabular}{lcccr}
\hline Treatment group & PreTX & $\mathbf{1 M}$ & 3M & $\mathbf{1 2 M}$ \\
\hline ATLG & $1.21+-0.28$ & $0.11+-0.07$ & $0.81+-0.43$ & $0.87+-0.43$ \\
Basiliximab & $1.27+-0.38$ & $0.74+-0.51$ & $1.87+-0.85$ & $1.90+-1.04$ \\
P & 0.795 & 0.051 & 0.048 & 0.093 \\
\hline
\end{tabular}

ATLG caused a lymphodepletion in the first months after transplantation.

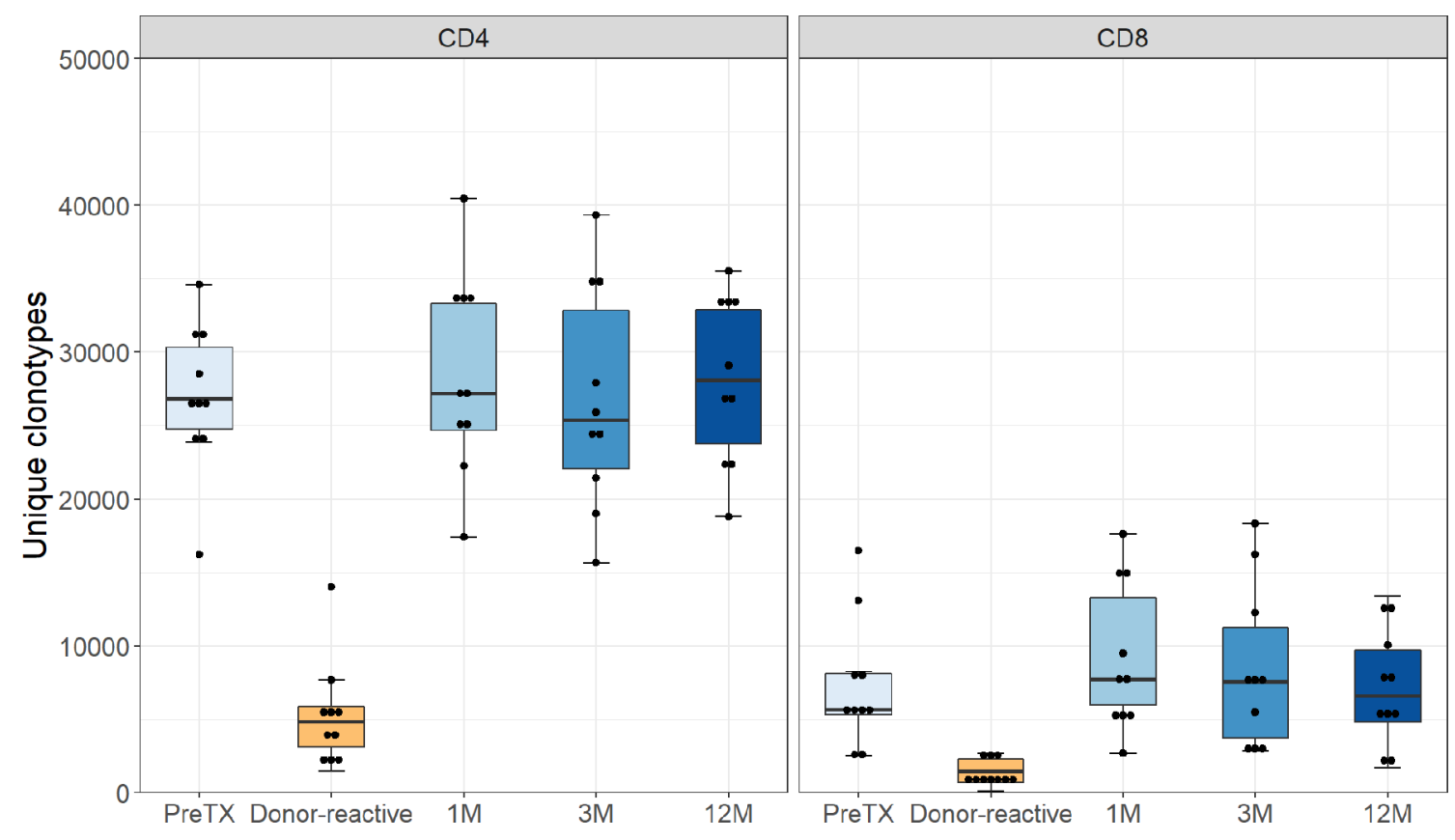

FIGURE 1 | Number of unique clonotypes after normalization by downsampling to the smallest number of reads. Grouped by phenotype groups CD4 and CD8 for the pre-transplant, the donor-reactive repertoire, and samples from the consecutive timepoints. The number of detected TCR clonotypes was comparable to pretransplant in both groups at all timepoints.

Phenotypic characterization of T-cells by FACS analysis in the group of pre-sensitized patients receiving ATLG treatment revealed a high heterogeneity of the patients in terms of their immune cell composition. Patients had decreased numbers of naive T-cells and elevated numbers of central memory CD4 Tcells prior to transplantation. The majority of patients showed a decline in recent thymic emigrants, indicating that reduced-dose ATLG was sufficient to achieve lymphodepletion in the thymus. An analysis of CD4 and CD8 T-cells revealed features consistent with lymphodepletional induction therapies, yet inversion of the CD4/CD8 ratio was, when observed at all, only minimal (Supplementary Figures 3-6).

\section{TCR Repertoire Characteristics}

The diversity of the TCR repertoires showed a wide individual variability. Between the two groups, there was no difference in clonality pre-transplant with a mean of 0.07 for CD4 and 0.31 for CD8 T-cells in the ATLG group and a mean of 0.04 for CD4 and 0.24 for CD8 T-cells in the basiliximab group. The clonality of post-transplant samples kept the individual variability and showed no statistical difference between the two groups over time in both CD4- and CD8-positive T-cells, respectively (CD4: $\mathrm{F}(1.45,11.6)=0.64 \mathrm{p}=0.496 ; \mathrm{CD} 8: \mathrm{F}(3,24)=0.60 \mathrm{p}=$ 0.620) (Figure 2).

The R20 measure, a metric for immune dominance, showed no segregation of the two cohorts indicating no increased clonal dominance in the overall TCR repertoire following reduced-dose ATLG treatment (Figure 3). The R20 of CD4- and CD8-positive T-cells after induction therapy was stable, and no statistical difference was observed in all timepoints after transplantation $(\mathrm{CD} 4: \mathrm{F}(3,24)=0.85 \mathrm{p}=0.479 ; \mathrm{CD} 8: \mathrm{F}(1.19,9.52)=0.79 \mathrm{p}=$ 0.418). A highly abundant clone in one subject in the ATLGtreated group (R294) represented more than $10 \%$ of the entire Tcell repertoire and was found in every timepoint pre- and posttransplant, responsible for the low R20 values in this patient.

We quantified if overall turnover in the TCR repertoire differed during lymphocyte reconstitution in the ATLG group compared to patients in the basiliximab group, which would be indicated by a high JSD. The measured JSD values were yet similar in the two groups at all timepoints 


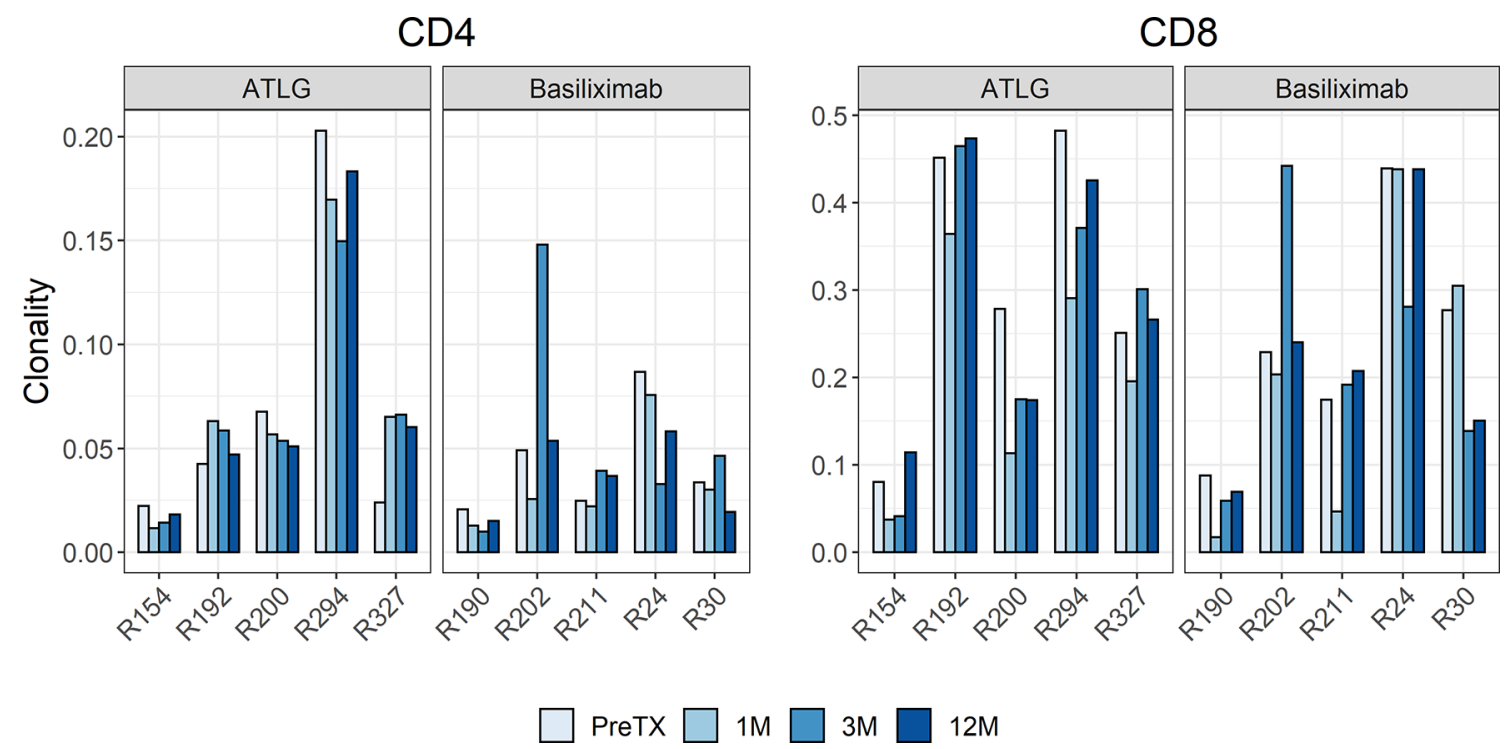

FIGURE 2 | Barchart of clonality estimates of the pre- and post-transplant repertoires grouped by CD4 and CD8 cells. The clonality of post-transplant samples showed no statistical difference between the ATLG and the basiliximab group.

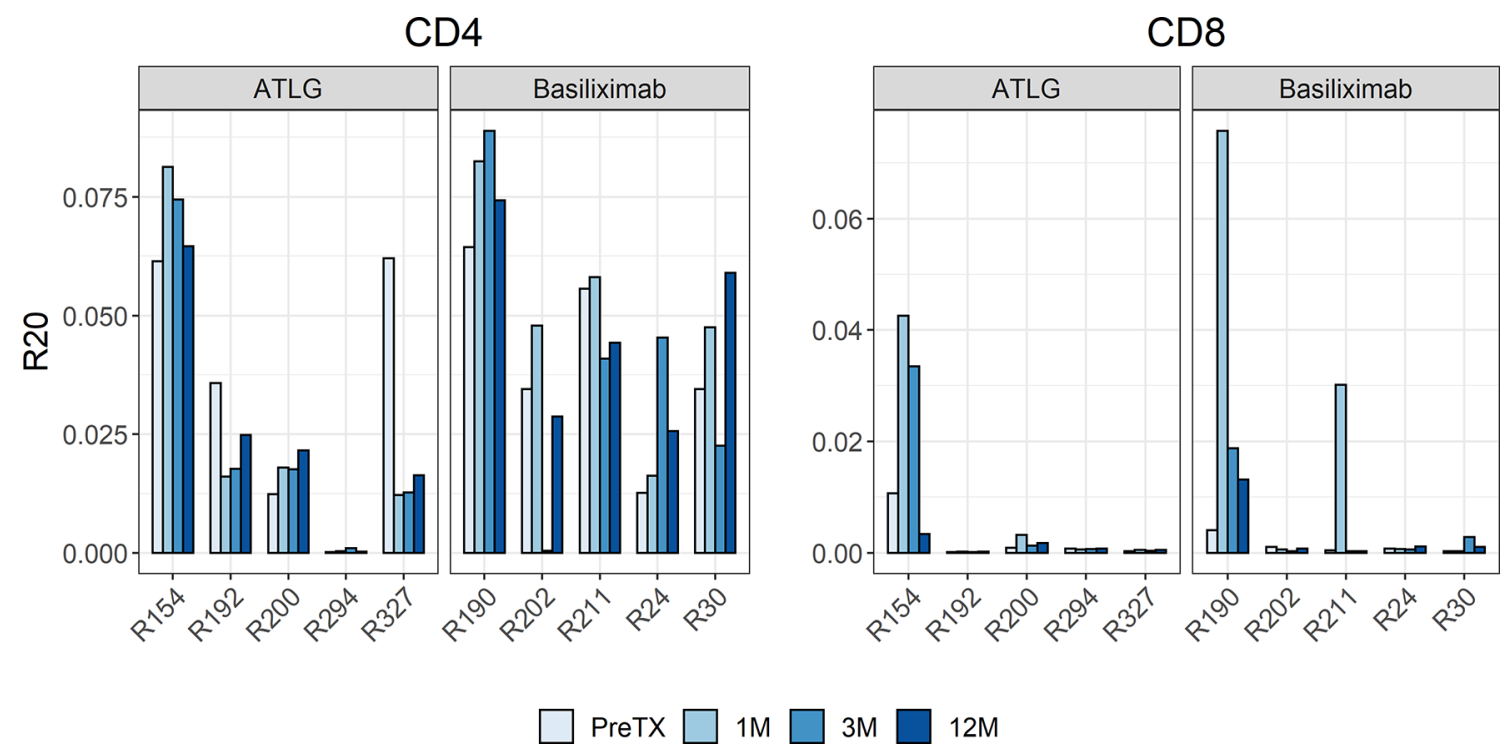

FIGURE 3 | Barchart of R20 estimates of the clonotypes in the pre- and post-transplant repertoires for CD4 and CD8 cells. The individual repertoire changes indicated no increased clonal dominance in the overall TCR repertoire following reduced-dose ATLG treatment.

post-transplant (Figure 4). This indicates that ATLG patients did not experience a higher repertoire turnover than basiliximab patients after transplantation.

Likewise, the change in VJ combination usage in the TCR repertoire pre- and post-induction treatment was similar between the two groups. Compared to the overall TCR repertoire, turnover divergence in VJ combination from baseline was low with a mean JSD of 0.02 and 0.07 for CD4 and $\mathrm{CD} 8$, respectively. VJ combination usage prior to transplantation and in the first month post-transplant from one representative patient in the ATLG induction group is shown in Figure 5. Quantification of TCRs with known specificities for Cytomegalovirus (CMV) or Epstein-Barr virus (EBV) both being responsible for common post-transplant viral infections showed that the number of virus-specific T-cells was comparable between the two treatment groups and remained 

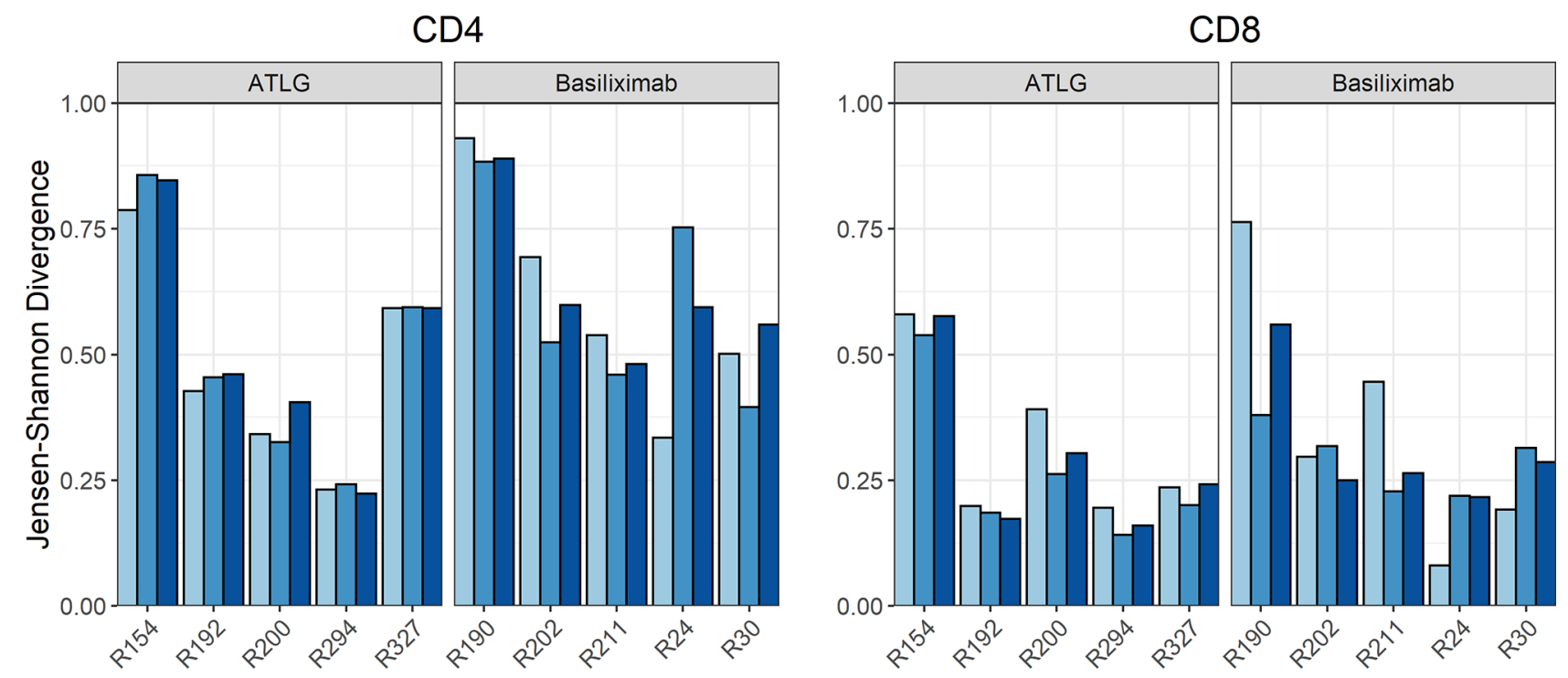

$1 \mathrm{M}$

$3 \mathrm{M}$

$12 \mathrm{M}$

FIGURE 4 | Time course of the Jensen-Shannon divergence (JSD) of the top 1,000 clones compared to baseline. No statistical difference was observed after transplantation, indicating that ATLG patients did not experience a higher repertoire turnover than basiliximab patients after transplantation.

stable over time (CMV: CD4: $\mathrm{F}(3,24)=0.76 \mathrm{p}=0.530 ; \mathrm{CD} 8: \mathrm{F}(3$, $24)=0.27 \mathrm{p}=0.849 ; \mathrm{EBV}: \mathrm{CD} 4: \mathrm{F}(3,24)=1.64 \mathrm{p}=0.206 ; \mathrm{CD} 8: \mathrm{F}$ $(1.13,9.03)=1.32 \mathrm{p}=0.287)$. Compared to the pre-transplant $\mathrm{T}$ cell repertoires, donor-reactive CD4 T-cell repertoires showed an increased abundance of CMV-specific T-cells $(\mathrm{t}(9)=-2.9 \mathrm{p}=$ 0.017). Cumulative frequencies of virus specific T-cells are provided in Supplementary Figure 7.

\section{The Donor-Reactive T-Cell Repertoire}

The preformed donor-reactive TCR repertoire was defined pretransplant. Analysis of preformed donor-reactive clonotypes detectable among all clonotypes in the bulk $\mathrm{T}$-cell repertoires pre- and post-transplant revealed a significant increase in the percentage of detectable CD4 donor-reactive clonotypes after transplantation irrespective of the induction therapy $(\mathrm{t}(9)=$ $-3.89 \mathrm{p}=0.004)$. In the ATLG group, the percentage of CD4 donor-reactive clonotypes increased on average by $0.67 \%$ to a total of $1.38 \% \pm 0.83$ after transplantation. Likewise, in the basiliximab group an average increase of $0.92 \%$ to a total of $1.64 \% \pm 1.17$ of CD4 donor-reactive clonotypes was observed after transplantation. The percentage of donor-reactive clonotypes in the bulk repertoires of all patients at all timepoints is visualized in Figure 6 and are shown in Supplementary Table 7. A numerical increase in donor-reactive clonotypes was seen in CD4 T-cells in all subjects in the basiliximab group over the observed time posttransplant, as expected. In the ATLG group, however, a less marked increase of donor-reactive cells in two individuals was of interest potentially caused by a prolonged lymphopenia in these subjects.
Contrary to the CD4 donor-reactive clonotypes, no statistically significant change in the percentage of CD8 donorreactive clonotypes was observed after transplantation $(\mathrm{t}(9)=$ $-1.01 \mathrm{p}=0.341)$.

Overall, the percentage of donor-reactive clonotypes was similar in both treatment groups throughout the posttransplant observation period $(\mathrm{CD} 4: \mathrm{F}(1.26,10.08)=1.38 \mathrm{p}=$ 0.279; CD8: $\mathrm{F}(1.06,8.48)=1.15 \mathrm{p}=0.319)$.

This increase of the donor-reactive repertoire was not only observed on a clonotype level but also on the level of frequencies of circulating donor-reactive T-cells after transplantation. For CD4 donor-reactive T-cells, a significant increase after transplantation was observed $(\mathrm{t}(9)=-3.59 \mathrm{p}=0.006)$, whereas the percentage of CD8 donor-reactive T-cells remained similar after transplantation $(\mathrm{t}(9)=1.57 \mathrm{p}=0.150)$. The mean increase of CD4 donor-reactive T-cells after transplantation in the ATLG and basiliximab group was $0.90 \%$ and $0.89 \%$, respectively.

\section{DISCUSSION}

In this study, we showed that reduced-dose ATLG induction therapy in sensitized kidney transplant recipients led to sufficient lymphodepletion without excessive clonal expansion of alloreactive T-cells. Excessive shifts in the overall diversity of the repopulating TCR repertoire were not seen and comparable to patients receiving basiliximab induction. Properties of the TCR repertoires, such as clonality and R20, exhibited between patient variability. This was, however, not unexpected as also a 


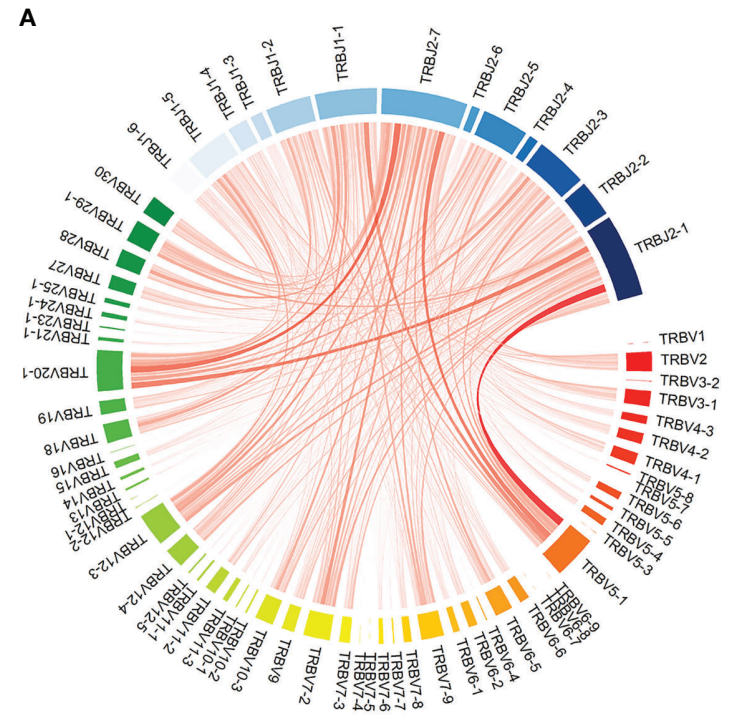

C

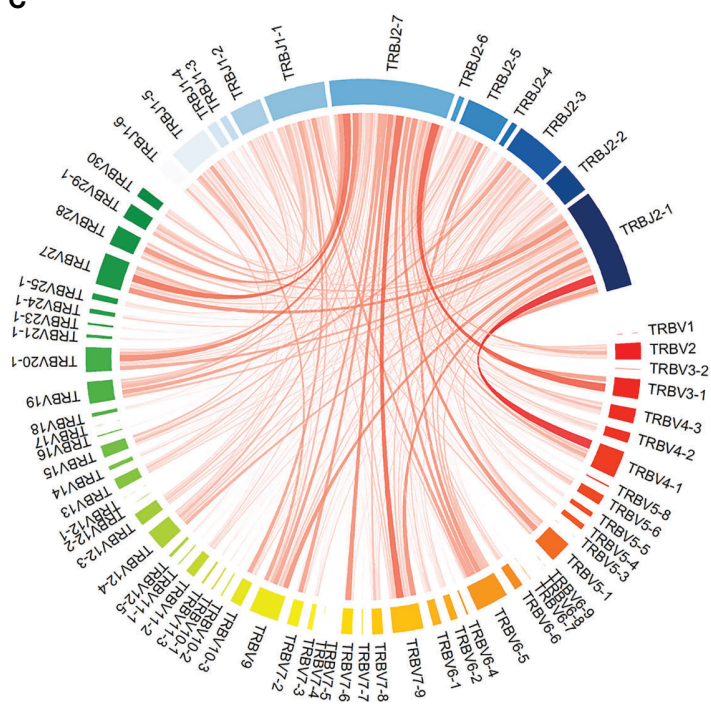

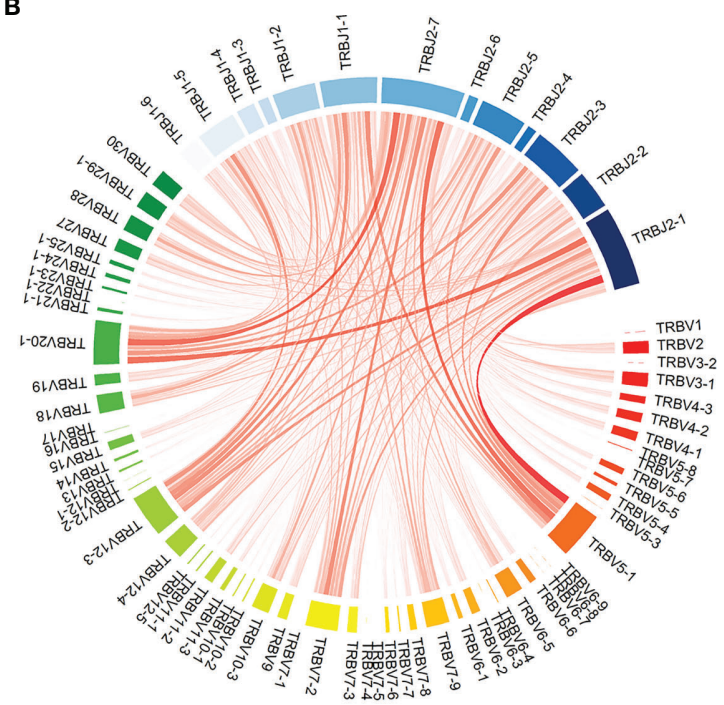

D

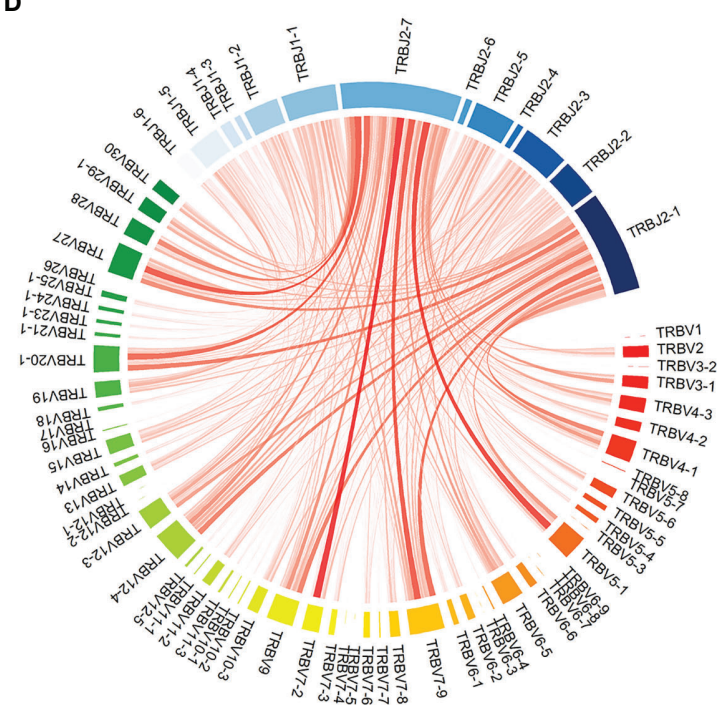

FIGURE 5 | Visualization of VJ combination usage in patient R154 from the reduced-dose ATLG induction group prior to transplantation and in the first month post transplantation. (A, D) show VJ combination usage in the full CD4 and CD8 TCR repertoires at the two timepoints: (A) PreTX CD4; (B) 1M CD4; (C) PreTX CD8; (D) $1 \mathrm{M}$ CD8.

previous study performed in healthy individuals observed interindividual differences in clonality and diversity that persisted over time (33). Such differences may reflect an individual's history of antigen exposure.

We detected an appearance of the donor-reactive T-cells already during the lymphopenic state as soon as 10 days after transplantation. Donor-reactive T-cells at similar rates were also present in patients receiving basiliximab, yet these patients present with normal lymphocyte counts after transplantation. The increase in donor-reactive T-cells in both groups did not affect the diversity of the overall TCR repertoire or repertoire turnover during or after lymphopenia resolved in the ATLG- treated subjects. This demonstrates that reduced-dose ATLG in pre-sensitized individuals leads to similar TCR repertoire changes as in non-sensitized basiliximab-treated patients with no inflation of CMV- or EBV-specific T-cells.

However, we observed an overrepresentation of CMVspecific $\mathrm{T}$-cells in the donor-reactive $\mathrm{T}$-cell repertoires. The existence of shared TCR sequences between CMV and donorreactive T-cells in HLA class I mismatched kidney transplant recipients has been described before (34).

At the phenotypic level, previously described changes after lymphodepletion were only observed to a lesser extent in this study. An expected skewed TCR repertoire and repopulation of 


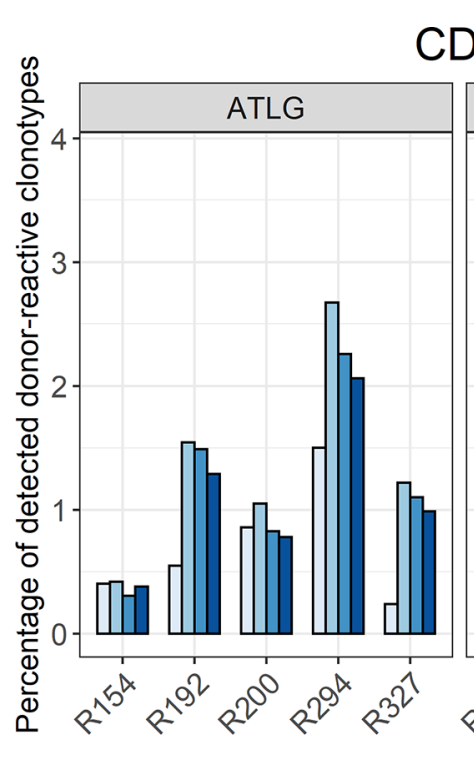

\section{CD4}

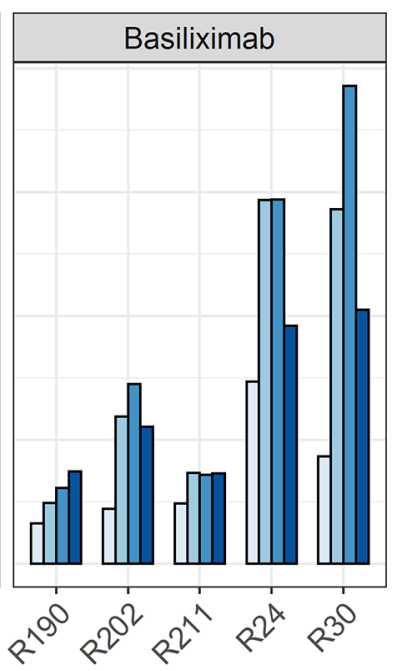

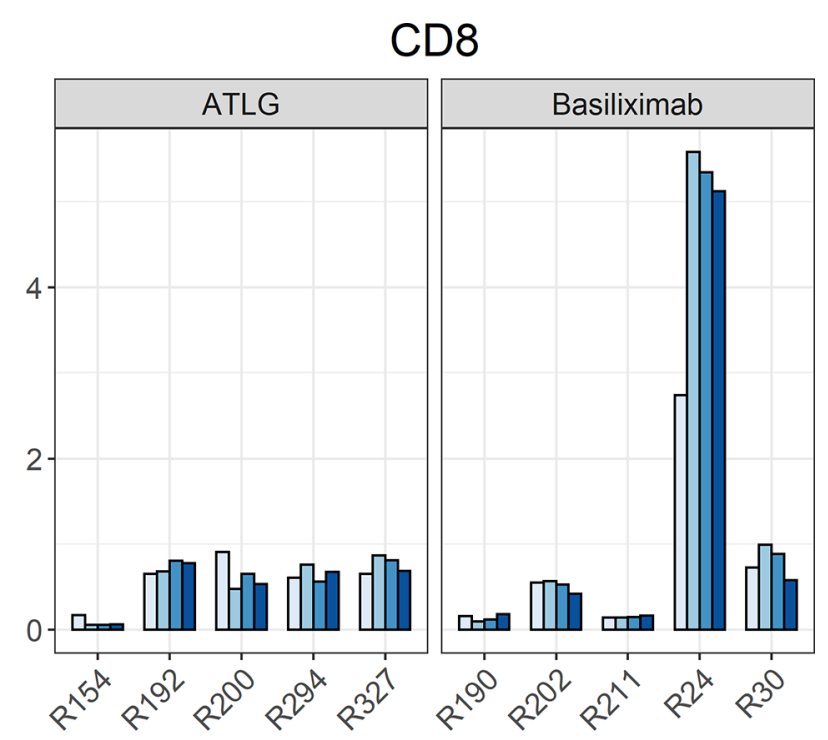

$3 \mathrm{M} \square 12 \mathrm{M}$

FIGURE 6 | Percentage of detected donor-reactive clonotypes after normalization by downsampling to the smallest number of reads. An increase in donor-reactive clonotypes early after transplantation was seen in the CD4 T-cell repertoire of the basiliximab group and the majority of patients in the ATLG group.

the peripheral lymphocyte repertoire by a restricted set of lymphocytes following lymphopenia was not observed after reduced-dose ATLG.

Previous studies described an inversion of the CD4/CD8 ratio, a more pronounced decrease of effector and naïve T-cells compared with cells of a memory phenotype, and a prolonged $\mathrm{T}$ cell dysfunction and reported a more severe and sustained lymphopenia $(5,11-18)$.

This is most likely due to higher doses, ranging from $4 \mathrm{mg} / \mathrm{kg}$ per day to up to $9 \mathrm{mg} / \mathrm{kg}$ at transplantation followed by $3 \mathrm{mg} / \mathrm{kg}$ for 4 days, of the lymphodepleting antibody, and the majority of studies have been conducted with ATG (Thymoglobuline ${ }^{\circledR}$ ), an anti-thymocyte globulin with different properties than ATLG. Thymoglobuline is produced by immunizing rabbits with fresh human thymocytes, and ATLG on the other hand is obtained by immunization of rabbits with Jurkat T-cells suggesting a narrower spectrum of targeted antigens by ATLG compared to Thymoglobuline $^{\circledR}$ (9). Comparable studies using reduced dosages of lymphodepletional antibodies were almost uniquely performed with Thymoglobuline, but likewise a lesser degree of T-cell depletion was observed with smaller doses (6, 35-37).

A limitation of this study is a small sample size; however, the detailed follow-up over 1 year is certainly sufficient to cover the range of immune reconstitution after lymphodepletion (no statistically significant difference in lymphocyte counts at the end of the observation period was observed between the groups), and all ATLG-treated patients were pre-sensitized and received identical maintenance immunosuppression.

The strength of this paper is the detailed characterization of lymphocyte repopulation following lymphodepletional induction therapy by TCR sequencing including not only the entire circulating TCR repertoire but also the donor-specific Tcells of each patient.

Based on the presented data, we conclude that the repopulated TCR repertoire in kidney transplant recipients after reduced-dose ATLG is not narrowed or skewed leading to comparable overall diversity and T-cell turnover as in patients receiving basiliximab. Likewise, donor-reactive $\mathrm{T}$-cells increase after a reduced-dose ATLG treatment in a similar way to basiliximab induced kidney transplant recipients.

Reduced-dose ATLG preserves the shape of the TCR repertoire, potentially leading to a lower susceptibility for infections or cancer without an increase of rejection episodes.

\section{DATA AVAILABILITY STATEMENT}

The datasets presented in this study can be found in online repositories. The names of the repository/repositories and accession number(s) can be found as follows: https://egaarchive.org, EGAD00001008478.

\section{ETHICS STATEMENT}

The study was reviewed and approved by the ethics committee of the Medical University of Vienna (EC NB: 267/2011). The patients provided their written informed consent to participate in this study. 


\section{AUTHOR CONTRIBUTIONS}

$\mathrm{RO}, \mathrm{AH}$, and $\mathrm{CA}$ were responsible for the conception, design, financial support, critical revision, and final approval of the manuscript. $\mathrm{RO}, \mathrm{CA}, \mathrm{KJ}$, and $\mathrm{AH}$ were responsible for the manuscript writing. KJ performed the library preparation for subsequent sequencing of all samples. KJ and MG prepared the figures. $\mathrm{MG}$ and $\mathrm{AH}$ were responsible for the statistics, bioinformatic analysis, and revision of the manuscript. $\mathrm{KH}$, $\mathrm{AH}$, and $\mathrm{CA}$ performed the sample collection, in vitro experiments, FACS sorting, and data analysis. RR-S, KH, SW, and TW were responsible for the critical revision of the manuscript. All authors contributed to the article and approved the submitted version.

\section{FUNDING}

The study was founded by the Scientific Funds of the Austrian National Bank [OeNb project number 17289 (https://www.oenb. at)], the WWTF (Vienna Science and Technology Fund, grant\#

\section{REFERENCES}

1. Ciancio G, Burke GW, Miller J. Induction Therapy in Renal Transplantation. Drugs (2007) 67(18):2667-80. doi: 10.2165/00003495-200767180-00003

2. Cravedi P, Mannon RB, Remuzzi G. Lymphocyte Depletion for Kidney Transplantation: Back to the Past? Nat Clin PractNephrology (2008) 4 (10):534-35. doi: 10.1038/ncpneph0914

3. Wiseman AC. Induction Therapy in Renal Transplantation: Why? What Agent? What Dose? We May Never Know. Clin J Am Soc Nephrol: CJASN (2015) 10(6):923-25. doi: 10.2215/CJN.03800415

4. Meier-Kriesche H-U, Arndorfer JA, Kaplan B. Association of Antibody Induction With Short- and Long-Term Cause-Specific Mortality in Renal Transplant Recipients. J Am Soc Nephrol (2002) 13(3):769-25. doi: 10.1681/ ASN.V133769

5. Weimer R, Ettrich M, Renner F, Dietrich H, Süsal C, Deisz S, et al. ATG Induction in Renal Transplant Recipients: Long-Term Hazard of Severe Infection Is Associated With Long-Term Functional T Cell Impairment But Not the ATG-Induced CD4 Cell Decline. Hum Immunol (2014) 75(6):561-69. doi: 10.1016/j.humimm.2014.02.015

6. Wong W, Agrawal N, Pascual M, Anderson DC, Hirsch HH, Fujimoto K, et al. Comparison of Two Dosages of Thymoglobulin Used as a Short-Course for Induction in Kidney Transplantation. Transplant International: Off J Eur Soc Organ Transplant (2006) 19(8):629-35. doi: 10.1111/j.1432-2277. 2006.00270.x

7. Klem P, Cooper JE, Weiss AS, Gralla J, Owen P, Chan L, et al. Reduced Dose Rabbit Anti-Thymocyte Globulin Induction for Prevention of Acute Rejection in High-Risk Kidney Transplant Recipients. Transplantation (2009) 88(7):891-96. doi: 10.1097/TP.0b013e3181b6f38c

8. Kho MML, Bouvy AP, Cadogan M, Kraaijeveld R, Baan CC, Weimar W. The Effect of Low and Ultra-Low Dosages Thymoglobulin on Peripheral T, B and NK Cells in Kidney Transplant Recipients. Transplant Immunol (2012) 26 (4):186-90. doi: 10.1016/j.trim.2012.02.003

9. Mohty M. Mechanisms of Action of Antithymocyte Globulin: T-Cell Depletion and Beyond. Leukemia (2007) 21(7):1387-94. doi: 10.1038/ sj.leu. 2404683

10. Ippoliti G, Lucioni M, Leonardi G, Paulli M. Immunomodulation With Rabbit Anti-Thymocyte Globulin in Solid Organ Transplantation. World J Transplant (2015) 5(4):261-66. doi: 10.5500/wjt.v5.i4.261

11. Weimer R, Staak A, Süsal C, Streller S, Yildiz S, Pelzl S, et al. ATG Induction Therapy: Long-Term Effects on Th1 But Not on Th2 Responses. Transplant
LS20-081), and the Medical University of Vienna Transplantation Research Platform's Start-Up Grant 2020. The funding bodies had no influence on the design, collection, analysis, and interpretation of data and writing the manuscript.

\section{ACKNOWLEDGMENTS}

We are grateful for the help in study administration to the study nurses of the Medical University of Vienna. We further want to thank the Core Facility Flow Cytometry, Medical University of Vienna, and the Core Facility Genomics, Medical University of Vienna, for providing the infrastructure for cell sorting and sequencing.

\section{SUPPLEMENTARY MATERIAL}

The Supplementary Material for this article can be found online at: https://www.frontiersin.org/articles/10.3389/fimmu.2022. 843452/full\#supplementary-material
International: Off J Eur Soc Organ Transplant (2005) 18(2):226-36. doi: 10.1111/j.1432-2277.2004.00047.x

12. Pearl JP, Parris J, Hale DA, Hoffmann SC, Bernstein WB, McCoy KL, et al. Immunocompetent T-Cells With a Memory-Like Phenotype Are the Dominant Cell Type Following Antibody-Mediated T-Cell Depletion. Am J Transplant: Off J Am Soc Transplant Am Soc Transplant Surgeons (2005) 5 (3):465-74. doi: 10.1111/j.1600-6143.2005.00759.x

13. Gurkan S, Luan Y, Dhillon N, Allam SR, Montague T, Bromberg JS, et al. Immune Reconstitution Following Rabbit Antithymocyte Globulin. Am J Transplant: Off J Am Soc Transplant Am Soc Transplant Surgeons (2010) 10 (9):2132-41. doi: 10.1111/j.1600-6143.2010.03210.x

14. Sewgobind DKD, Kho MML, van der Laan LJW, Hendrikx TK, van Dam T, Tilanus HW, et al. The Effect of Rabbit Anti-Thymocyte Globulin Induction Therapy on Regulatory T Cells in Kidney Transplant Patients. Nephrol Dialysis Transplant: Off Publ Eur Dialysis Transplant Assoc - Eur Renal Assoc (2009) 24(5):1635-44. doi: 10.1093/ndt/gfn778

15. Tang Q, Leung J, Melli K, Lay K, Chuu EL, Liu W, et al. Altered Balance Between Effector T Cells and FOXP3+ HELIOS+ Regulatory T Cells After Thymoglobulin Induction in Kidney Transplant Recipients. Transplant International: Off J Eur Soc Organ Transplant (2012) 25(12):1257-67. doi: 10.1111/j.1432-2277.2012.01565.x

16. Longuet H, Sautenet B, Gatault P, Thibault G, Barbet C, Marliere J-F, et al. Risk Factors for Impaired CD4+ T-Cell Reconstitution Following Rabbit Antithymocyte Globulin Treatment in Kidney Transplantation. Transplant International: Off J Eur Soc Organ Transplant (2014) 27(3):271-795. doi: $10.1111 /$ tri.12249

17. Louis S, Audrain M, Cantarovich D, Schaffrath B, Hofmann K, Janssen U, et al. Long-Term Cell Monitoring of Kidney Recipients After an Antilymphocyte Globulin Induction With and Without Steroids. Transplantation (2007) 83(6):712-21. doi: 10.1097/01.tp.0000255683. 66156.d3

18. Crepin T, Carron C, Roubiou C, Gaugler B, Gaiffe E, Simula-Faivre D, et al. ATG-Induced Accelerated Immune Senescence: Clinical Implications in Renal Transplant Recipients. Am J Transplant: Off J Am Soc Transplant Am Soc Transplant Surgeons (2015) 15(4):1028-38. doi: 10.1111/ajt.13092

19. Cherkassky L, Lanning M, Lalli PN, Czerr J, Siegel H, Danziger-Isakov L, et al. Evaluation of Alloreactivity in Kidney Transplant Recipients Treated With Antithymocyte Globulin Versus IL-2 Receptor Blocker. Am J Transplant: Off J Am Soc Transplant Am Soc Transplant Surgeons (2011) 11(7):1388-96. doi: 10.1111/j.1600-6143.2011.03540.x 
20. DeWolf S, Grinshpun B, Savage T, Ping Lau S, Obradovic A, Shonts B, et al. Quantifying Size and Diversity of the Human T Cell Alloresponse. JCI Insight (2018) 3(15). doi: 10.1172/jci.insight.121256

21. Shugay M, Britanova OV, Merzlyak EM, Turchaninova MA, Mamedov IZ, Tuganbaev TR, et al. Towards Error-Free Profiling of Immune Repertoires. Nat Methods (2014) 11(6):653-55. doi: 10.1038/nmeth.2960

22. Morris H, DeWolf S, Robins H, Sprangers B, LoCascio SA, Shonts BA, et al. Tracking Donor-Reactive T Cells: Evidence for Clonal Deletion in Tolerant Kidney Transplant Patients. Sci Trans Med (2015) 7(272):272ra10. doi: 10.1126/scitranslmed.3010760

23. Aschauer C, Jelencsics K, Hu K, Heinzel A, Gregorich MG, Vetter J, et al. Prospective Tracking of Donor-Reactive T-Cell Clones in the Circulation and Rejecting Human Kidney Allografts. Front Immunol (2021) 12:750005. doi: $10.3389 /$ fimmu.2021.750005

24. Fu J, Zuber J, Martinez M, Shonts B, Obradovic A, Wang H, et al. Human Intestinal Allografts Contain Functional Hematopoietic Stem and Progenitor Cells That Are Maintained by a Circulating Pool. Cell Stem Cell (2019) 24 (2):227-239.e8. doi: 10.1016/j.stem.2018.11.007

25. Zuber J, Brittany S S-P, Obradovic A, Fu J, Yang S, Lambert M, et al. Bidirectional Intragraft Alloreactivity Drives the Repopulation of Human Intestinal Allografts and Correlates With Clinical Outcome. Sci Immunol (2016) 1(4). doi: 10.1126/sciimmunol.aah 3732

26. Faé I, Wenda S, Grill C, Fischer GF. HLA-B*44:138Q: Evidence for a Confined Deletion and Recombination Events in an Otherwise Unaffected HLAHaplotype. HLA (2019) 93(2-3):89-96. doi: 10.1111/tan.13439

27. Schwaiger E, Eskandary F, Kozakowski N, Bond GR, Kikić Ž, Yoo D, et al. Deceased Donor Kidney Transplantation Across Donor-Specific Antibody Barriers: Predictors of Antibody-Mediated Rejection. Nephrol Dialysis Transplant: Off Publ Eur Dialysis Transplant Assoc - Eur Renal Assoc (2016) 31(8):1342-515. doi: 10.1093/ndt/gfw027

28. DeWolf S, Sykes M. Alloimmune T Cells in Transplantation. J Clin Invest (2017) 127(7):2473-81. doi: 10.1172/JCI90595

29. Aschauer C, Jelencsics K, Hu K, Heinzel A, Vetter J, Fraunhofer T, et al. Next Generation Sequencing Based Assessment of the Alloreactive T Cell Receptor Repertoire in Kidney Transplant Patients During Rejection: A Prospective Cohort Study. BMC Nephrol (2019) 20(1):346. doi: 10.1186/s12882-019-1541-5

30. Bolotin DA, Poslavsky S, Mitrophanov I, Shugay M, Mamedov IZ, Putintseva EV, et al. MiXCR: Software for Comprehensive Adaptive Immunity Profiling. Nat Methods (2015) 12(5):380-815. doi: 10.1038/nmeth.3364

31. Shugay M, Bagaev DV, Zvyagin IV, Vroomans RM, Crawford JC, Dolton G, et al. VDJdb: A Curated Database of T-Cell Receptor Sequences With Known Antigen Specificity. Nucleic Acids Res (2018) 46(D1):D419-27. doi: 10.1093/nar/gkx760
32. Chaudhary N, Wesemann DR. Analyzing Immunoglobulin Repertoires. Front Immunol (2018) 9:462. doi: 10.3389/fimmu.2018.00462

33. Chu ND, Bi HS, Emerson RO, Sherwood AM, Birnbaum ME, Robins HS, et al. Longitudinal Immunosequencing in Healthy People Reveals Persistent T Cell Receptors Rich in Highly Public Receptors. BMC Immunol (2019) 20:19. doi: $10.1186 / \mathrm{s} 12865-019-0300-5$

34. Stranavova L, Pelak O, Svaton M, Hruba P, Fronkova E, Slavcev A, et al. Heterologous Cytomegalovirus and Allo-Reactivity by Shared T Cell Receptor Repertoire in Kidney Transplantation. Front Immunol (2019) 10:3389/ fimmu.2019.02549. doi: 10.3389/fimmu.2019.02549

35. Ruggenenti P, Codreanu I, Cravedi P, Perna A, Gotti E, Remuzzi G. Basiliximab Combined With Low-Dose Rabbit Anti-Human Thymocyte Globulin: A Possible Further Step Toward Effective and Minimally Toxic T Cell-Targeted Therapy in Kidney Transplantation. Clin J Am Soc Nephrol: CJASN (2006) 1(3):546-54. doi: 10.2215/CJN.01841105

36. Préville X, Flacher M, LeMauff B, Beauchard S, Davelu P, Tiollier J, et al. Mechanisms Involved in Antithymocyte Globulin Immunosuppressive Activity in a Nonhuman Primate Model 1. Transplantation (2001) 71 (3):460-68. doi: 10.1097/00007890-200102150-00021

37. Stevens RB, Foster KW, Miles CD, Lane JT, Kalil AC, Florescu DF, et al. A Randomized 2×2 Factorial Trial, Part 1: Single-Dose Rabbit Antithymocyte Globulin Induction May Improve Renal Transplantation Outcomes. Transplantation (2015) 99(1):197-209. doi: 10.1097/TP.0000000000000250

Conflict of Interest: The authors declare that the research was conducted in the absence of any commercial or financial relationships that could be construed as a potential conflict of interest.

Publisher's Note: All claims expressed in this article are solely those of the authors and do not necessarily represent those of their affiliated organizations, or those of the publisher, the editors and the reviewers. Any product that may be evaluated in this article, or claim that may be made by its manufacturer, is not guaranteed or endorsed by the publisher.

Copyright $\odot 2022$ Aschauer, Jelencsics, Hu, Gregorich, Reindl-Schwaighofer, Wenda, Wekerle, Heinzel and Oberbauer. This is an open-access article distributed under the terms of the Creative Commons Attribution License (CC BY). The use, distribution or reproduction in other forums is permitted, provided the original author(s) and the copyright owner(s) are credited and that the original publication in this journal is cited, in accordance with accepted academic practice. No use, distribution or reproduction is permitted which does not comply with these terms. 